\title{
Aerodynamic Performance of an Ultra-Low Aspect Ratio Centripetal Turbine Stator ${ }^{\dagger}$
}

\author{
Fabrizio Fontaneto ${ }^{1, *}$, Tony Arts ${ }^{1}$, Matthieu Simon ${ }^{2}$ and Philippe Picot ${ }^{2}$ \\ 1 Jacques Chauvin Laboratory, von Karman Institute for Fluid Dynamics, Waterloosesteenweg 72, \\ B-1640 Sint-Genesius-Rode, Belgium; arts@vki.ac.be \\ 2 Safran Aircraft Engines, Rond Point René Ravaud-Réau, 77550 Moissy-Cramayel, France; \\ matthieu.simon@safrangroup.com (M.S.); philippe.picot@safrangroup.com (P.P.) \\ * Correspondence: fontaneto@vki.ac.be; Tel.: +32-2-359-9630 \\ $+\quad$ This paper is an extended version of our paper published in Proceedings of the European Turbomachinery \\ Conference ETC'11, 2015, Paper No. 18.
}

Academic Editor: Marcello Manna

Received: 26 August 2016; Accepted: 18 November 2016; Published: 26 November 2016

\begin{abstract}
An extensive measurement campaign was carried out at the von Karman Institute for Fluid Dynamics to assess the aerodynamic performance of an ultra-low aspect ratio centripetal turbine stator. The test section consisted of 18 periodic sectors of two blade passages each, tested at transonic conditions in a blow-down facility. Particle image velocimetry (2D-PIV) measurements were performed in the trailing edge area at blade mid-height. Further downstream, 16 micro virtual 3 -hole pressure probes were used to measure the aerodynamic performance at about $44 \%$ of the radial chord, downstream of the trailing edge. Results describe a highly swirling flow-field characterized by a very large tangential velocity component increasing at smaller radii. The radial component decreased instead to compensate for an opening of the test section closer to its center, therefore enhancing the tangential nature of the generated flow field. Adjacent blades showed asymmetric wakes as an effect of the particular design of the nozzle. The performance analysis exhibited very high loss coefficients (always higher than 12\%) which have to be related to the extremely small aspect ratio of the cascade.
\end{abstract}

Keywords: ultra-low aspect ratio; centripetal turbine stator; transonic; micro pressure probe; virtual 3-hole; particle image velocimetry; aerodynamic performance

\section{Introduction}

In the last decades, the typical design targets for new aero-engine architectures always involved an increase of the efficiency and of the power output of the thermodynamic cycle by maximizing the turbine inlet temperature. As a consequence, the heat load to the high pressure turbine (HPT) rose as well, forcing the use of a colder cooling flow in order to meet the required thermal protection needs. A reduction of the coolant temperature can be reached by means of an expansion along the coolant delivery circuit, before feeding the blade and the disk area. Consequently, after flowing over the disk surfaces, the cooling air is then injected into the mainstream from the inter-platform gap where it is further used to protect the leading edge region of the endwall. In the latter application in particular, a high compatibility with the flow angles of the main stream is also recommended to reduce the aerodynamic impact of the injection as much as possible.

A possible design which meets all these requirements consists in a centripetal turbine stator located in the core of the engine, at a smaller radius than the endwall (see Figure 1). This configuration, moreover, guarantees a high degree of compactness of the component, leading to the adoption of ultra-low aspect ratio blades. 


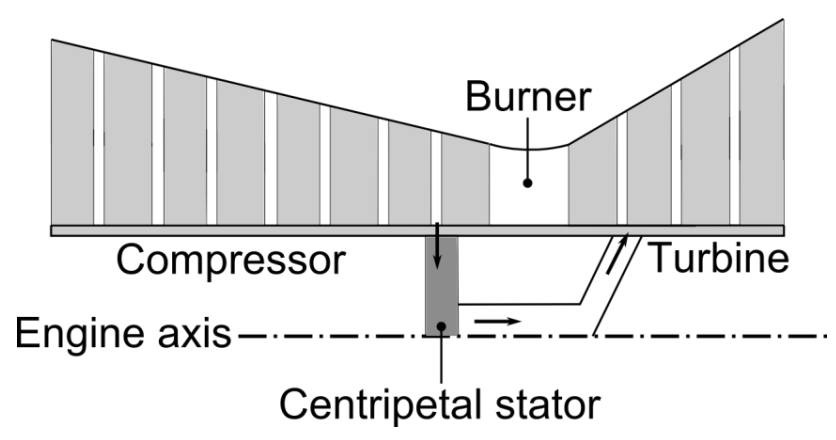

Figure 1. Typical engine arrangement of a centripetal turbine stator.

The development of such a component faces a deep lack of publication in the open literature. The most complete contribution on this topic available today was provided by Simpson et al. [1] who performed an extensive numerical study describing the effect of solidity and vaneless space on the performance of a low aspect ratio automotive radial turbine stator. This experimental activity was limited to the measurement of the downstream wall static pressure distribution which was then used to evaluate the quality of the numerical predictions. Results were given mainly in terms of mass-averaged kinetic energy loss coefficient $(\zeta)$ and showed that the solidity had the strongest effect on the stator efficiency: $\zeta$ grew almost linearly with the number of blades of the cascade, leading to values as high as $20 \%$ for the 30 blades stator configuration.

An extensive measurement campaign was recently carried out at the von Karman Institute for Fluid Dynamics (VKI) on a transonic ultra-low aspect ratio centripetal turbine stator with the intent of providing a detailed insight into the phenomenology of the flow field that such a component generates. Total pressure, velocity, and flow angle distributions were measured at two different radial locations downstream of the nozzle. Near to the trailing edge, the performance of the cascade was assessed by particle image velocimetry (PIV) measurements which were then processed with an advanced PIV processor. Further downstream, the total pressure and flow angle distributions were measured by means of 16 micro virtual 3-hole probes.

In the present contribution, the test section is described and the measurement techniques discussed, paying a particular attention to the issues related to the challenging geometrical constraints that directly drove and affected the design of the experiments. Results are successively presented and critically analyzed in terms of total pressure wakes, flow angle and loss coefficient distributions at two different radial positions in the discharge section of the cascade.

\section{Test Section, Instrumentation, and Test Conditions}

The test section consisted of 18 periodic sectors $20^{\circ}$ wide. As is seen in Figure 2 (schematic view not to scale in the test section), each sector consisted of a "clean" and a "covered" blade. The "clean" blade had the leading edge aligned with the mean line of the upstream feeding hole, while the inlet section of the "covered" one was, instead, blocked by a wall for fixing purposes. According to the sketch of the meridional flow path presented in Figure 3, downstream the race-track hole a settling volume was placed, which fed the bladed part of the test section. The latter was only $6.8 \mathrm{~mm}$ high to become $8.4 \mathrm{~mm}$ further downstream of the trailing edge radius, where an opening was present. The facility is a typical blow-down wind tunnel: the flow was drawn from a pressurized reservoir and radially delivered to the upstream distribution annulus at temperatures ranging between $10^{\circ}$ and $15^{\circ}$. After flowing through the cascade, it was then discharged axially at atmospheric conditions. Tests were carried out at full Mach number (Ma) similarity with engine conditions, while the effect of the lower total inlet temperature on the Reynolds number (Re) was partially counter-balanced by the use of a scaled model, which allowed the retrieval of Re values of the same order of magnitude as the engine ones, sufficiently high to consider a turbulent flow. 


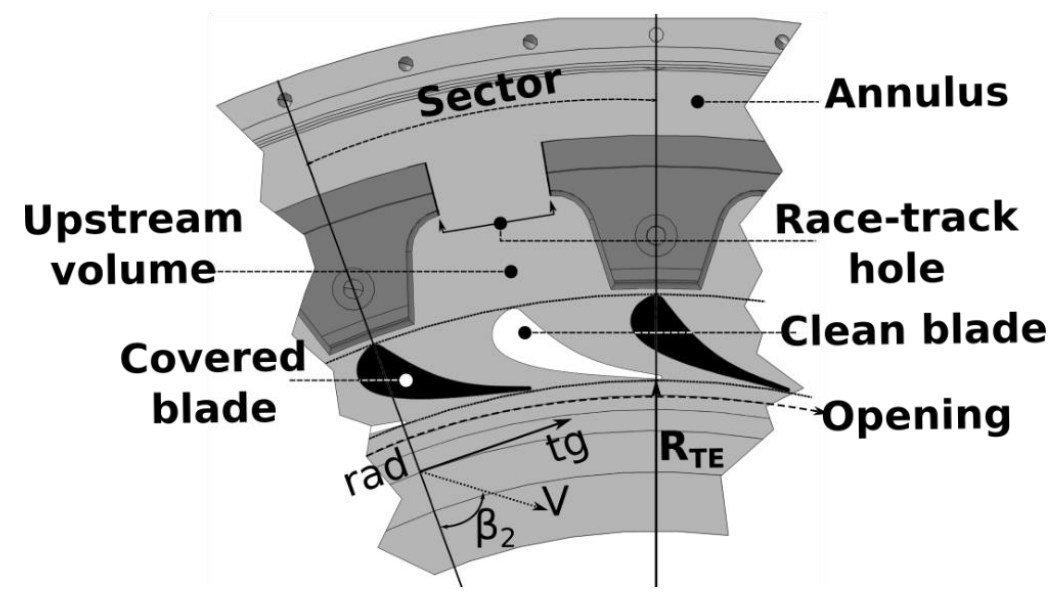

Figure 2. Frontal view of a single sector of the test section: adopted conventions and nomenclature. $\mathrm{R}_{\mathrm{TE}}$ : trailing edge radius; rad: radial direction; tg: tangential direction; $\mathrm{V}$ : absolute velocity; $\beta_{2}$ : stator discharge angle.

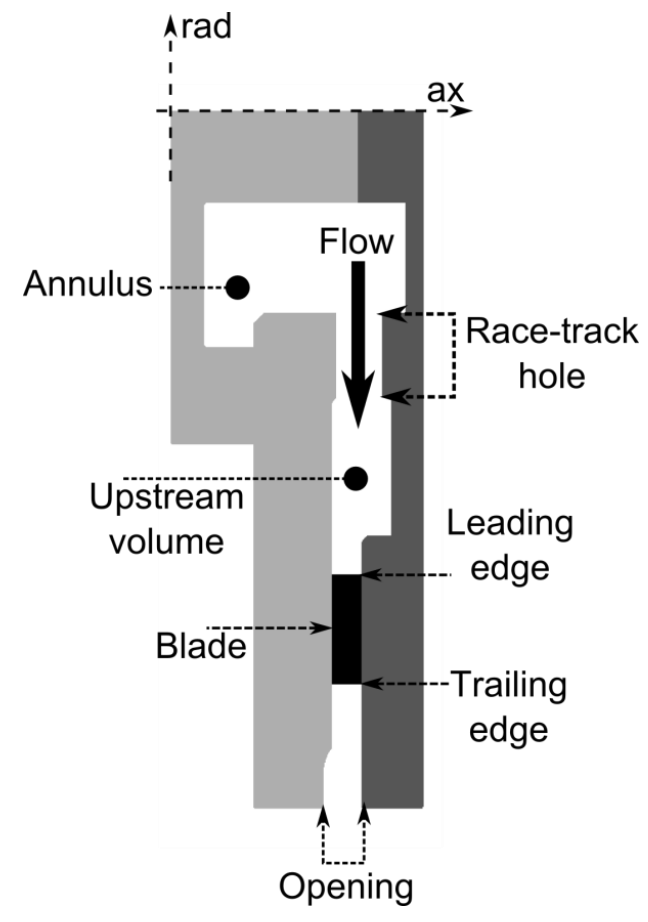

Figure 3. Principle sketch of the meridional flow path (not to scale). Rad: radial direction; ax: axial direction.

The test section was sealed from the exterior by means of a carrier which was directly carrying "on-board" the optical access window for PIV measurements, as well as all of the rest of the instrumentation, as shown in Figure 4 . The homogeneity of the total inlet pressure $\mathrm{P}_{01}$ was checked by means of 10 total pressure probes located at $31.7 \%$ of the radial chord $\left(C_{r}\right)$ upstream of the leading edge plane and distributed over many different sectors. At the same radial location, one sector was equipped with a rack of nine static pressure taps to retrieve the azimuthal wall static pressure distribution $P_{1}$. The downstream wall static pressure $P_{2}$ was measured by means of two rows of 25 static pressure taps located, respectively, at $8.6 \% \mathrm{C}_{\mathrm{r}}$ and $44.5 \% \mathrm{C}_{\mathrm{r}}$ downstream of the trailing edge, covering 1.5 sectors in total. The downstream total pressure $\left(\mathrm{P}_{02}\right)$ distribution was also measured at $44.5 \% \mathrm{C}_{\mathrm{r}}$ by 16 micro cylindrical pressure probes operated in a virtual 3-hole mode. The probes 
were distributed circumferentially over several sectors in such a way that they covered an entire sector (i.e., two adjacent blade passages). Tests were carried out at nearly ambient inlet total temperature and at a target isentropic discharge Mach number $\left(\mathrm{Ma}_{2 \mathrm{~s}}\right)$ of 0.9 .

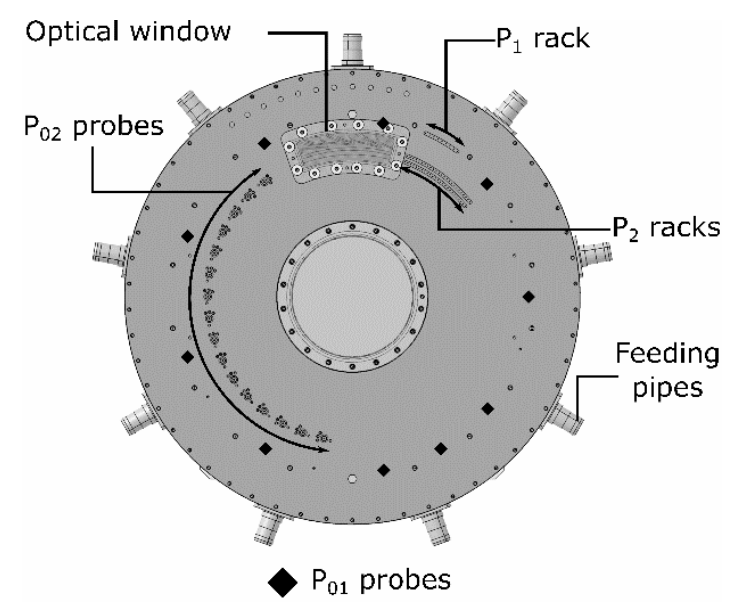

Figure 4. View of the instrumented carrier.

\section{Experimental Methodology}

\subsection{Particle Image Velocimetry}

The PIV measurement setup is shown in Figure 5. The laser beam, provided by a Quantel pulsed Twin CFR Nd:YAG laser (532 nm) light source (Quantel Laser, Les Ulis Cedex, France), was turned into the direction of the test section axis by a right angle prism. A cylindrical lens was used to open the beam into a laser sheet which was then focused by a $500 \mathrm{~mm}$ focal length lens. A second right angle prism was then fitted into the facility to turn the laser sheet parallel to the cascade endwall. The resulting PIV measurement plane was set at the blade's mid-height; the light sheet thickness was about $1 \mathrm{~mm}$ in the trailing edge region. PIV images were recorded by means of a PCO 1.3 megapixel Sensycam CCD camera (PCO, Kelheim, Germany), fitted with a $50 \mathrm{~mm}$ NIKKOR objective (Nikon, Melville, NY, USA). The limited access provided by the optical Plexiglass window led to a PIV interrogation area restricted to the suction side of a covered blade, its corresponding throat region, and part of the downstream section, as shown in Figure 6.

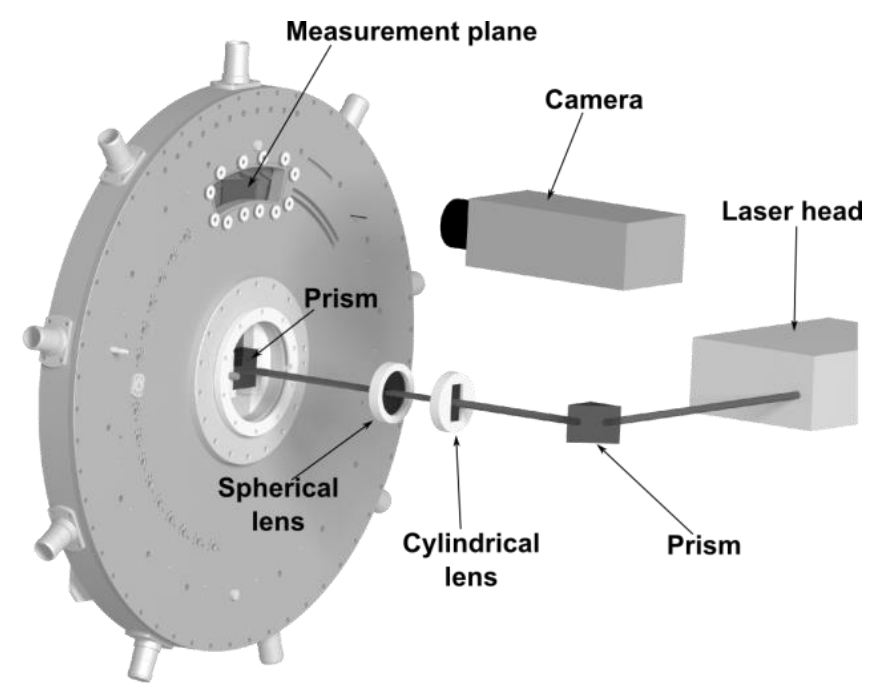

Figure 5. Particle Image velocimetry (PIV) optical setup arrangement. 


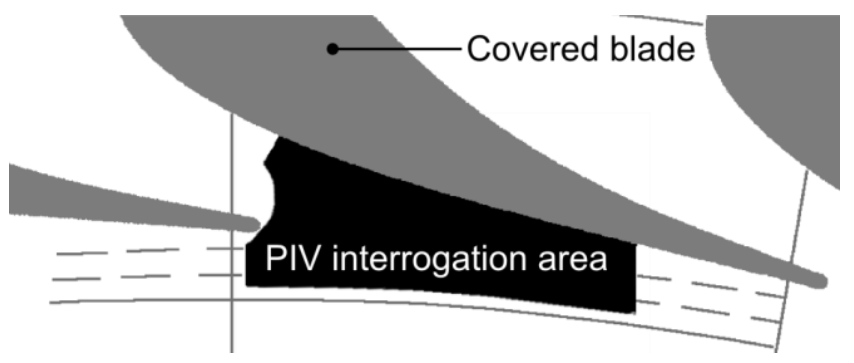

Figure 6. Field of view of PIV measurements.

Since the tests were carried out at transonic conditions, a separation time of $0.78 \mu \mathrm{s}$ was adopted. Incense smoke was used as tracer as it contains particles of about $1 \mu \mathrm{m}$ in size [2]. The smoke was provided from a pressurized vessel, equipped with two hotplates used to burn commercially available granulated incense. The injection point was located upstream the leading edge plane, in the "trapezoidal" volume, where a total pressure probe was also present to verify the homogeneity of the inlet conditions with respect to the other sectors.

During the normal test time lapse, the temperature of the wetted surfaces of the facility drastically reduced because of the total inlet temperature drop (typical of every blow-down facility) and of the flow static temperature reduction imposed by the expansion. In these conditions, the small fraction of water vapor suspended in the flow close to the endwalls condensed and generated a variation of the reflections pattern in time. Moreover, the thin condensed layer was acting as a trap for the small incense particles, leading to the entrainment of some of them by the endwall roughness and, therefore, to the generation of stationary bright spots in the background. Given the extremely short distance between the PIV measurement plane and the endwall (about $3 \mathrm{~mm}$ ), the stationary spots were also recorded by the camera and directly generated a zero displacement vector at the smallest aperture. In order to get rid of these types of artifacts, images were pre-processed by means of an in-house code that performed a pixel-wise minimum intensity subtraction on each image pair individually. Stationary particles and variable reflections effects could, therefore, be efficiently removed.

The PIV measurements were performed in a region characterized by a high vorticity level and strong velocity gradients. In these conditions, standard commercial PIV codes demonstrated to be inefficient as shown by Fontaneto et al. [3]. Therefore, in order to retrieve reliable velocity fields, the advanced adaptive PIV processor ARPIV developed by Theunissen [4] was used. For each image pair, the code first performs a particle recognition analysis. Interrogation windows are then displaced all over the field of view, providing to maintain a minimum number of particles as set by the operator. The code refines, through successive iterations, the position and the size of the interrogation cells, adapting them according to the displacement vector computed at the previous step but also maintaining a high number of particles for each cell.

\subsection{Micro Virtual 3-Hole Probes}

A set of 16 micro cylindrical probes was placed downstream of the trailing edge, at $44.5 \% \mathrm{C}_{\mathrm{r}}$. The probes were distributed over several sectors (see Figure 4) in such a way to cover a full sector periodicity with an angular step of $1.25^{\circ}$. As shown in Figure 7 , the measuring head of the probe, the only part exposed to the flow, consisted of a $0.6 \mathrm{~mm}$ external diameter pipe with a $0.1 \mathrm{~mm}$ drilled pressure tap. Considering the global frontal area of the probes, the latter corresponded to $0.3 \%$ of the flow cross-section, therefore, inducing a negligible blockage effect on the flow field. This head was welded to a fixing base which guaranteed a univocal positioning from the exterior of the facility by means of a fixing pin. 


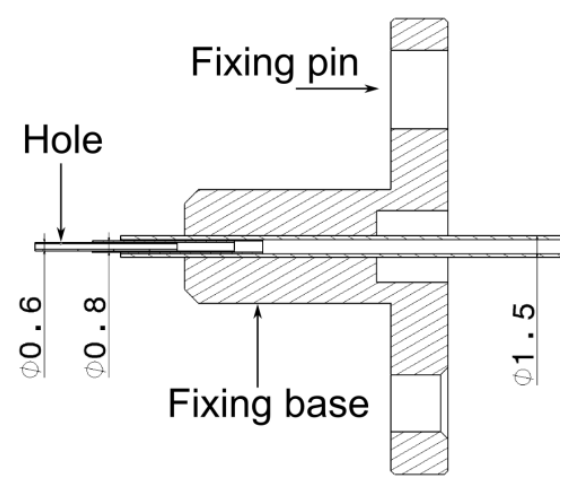

Figure 7. Cut-view of the micro virtual 3-hole pressure probes.

The probes were operated in a virtual 3-hole mode, allowing to retrieve the downstream flow angle $\left(\beta_{2}\right)$ and total pressure distributions. This was performed by rotating the probes in three different positions with an angular step of $35^{\circ}$ and by applying an angular calibration previously performed. Even though it was possible to modify the angular position of the probes without opening the test section, the facility had anyway to be switched off inducing a slight variation of the upstream total pressure between the three different angular positioning. In order, therefore, to enhance the quality of the results, all pressure measurements were normalized with respect to a reference inlet total pressure value prior to reducing the data. The complete normalization procedure is described by Fontaneto et al. in [3], where further details about the angular calibration of the micro cylindrical probes are also provided.

Given the very small dimensions of the pressure tap, the dynamic response of probes was checked with the intent of defining the correct settling time to be due before the start of the acquisition. The transfer function of such a pneumatic system was evaluated by means of the model of Bergh and Tijdeman [5], considering typical upstream total conditions. The result showed a classical first order system behavior whose time constant was found to be about $0.2 \mathrm{~s}$ at the $3 \mathrm{~dB}$ cutoff frequency. Since the used model necessarily introduces some degrees of approximation it was, in the end, decided to extend it to $2 \mathrm{~s}$.

\section{Inlet Homogeneity and Outlet Periodicity}

As mentioned previously, the testing air was provided to every sector by means of a large-volume feeding annulus which guaranteed a high degree of uniformity of the inlet conditions. This is confirmed by the polar plot of Figure 8 where the relative difference between the local measured total pressures and the global mean value never exceeds $\pm 0.3 \%$. The $\mathrm{P}_{01}$ distribution was measured by means of 10 probes displaced over as many sectors and aligned with the leading edge (LE) of the "clean" blade. At the same radial coordinate, nine static pressure taps were then distributed along the inlet trapezoidal cavity of a typical sector (see Figure 4). The resulting $P_{1}$ distribution is shown in Figure 9 and it is characterized by a flat evolution in the central region (centered with the "clean" blade) which drops by about $1 \%$ at the sides, close to the "covered" blades' inlet areas.

The periodicity of the flow in the discharge section was verified by means of two rows of 25 static pressure taps each and respectively located at $8.6 \% \mathrm{C}_{\mathrm{r}}$ and $44.5 \% \mathrm{C}_{\mathrm{r}}$ downstream of the trailing edge. The pitch between the taps was such that 1.5 sectors could be measured. The corresponding isentropic discharge Mach number $\left(\mathrm{Ma}_{2 \mathrm{~s}}\right)$ is plotted in Figure 10 against the azimuthal coordinate and describes a marked free vortex behavior of the flow field generated in this region: while flowing towards the outlet channel, at the center of the test section, the flow is further accelerated, reaching an almost sonic condition on row 2 . While flowing from row 1 to 2 , the distribution becomes more symmetrical and homogeneous even though a high degree of periodicity could always be recognized, with no evident differences between "covered" and "clean" blades. 


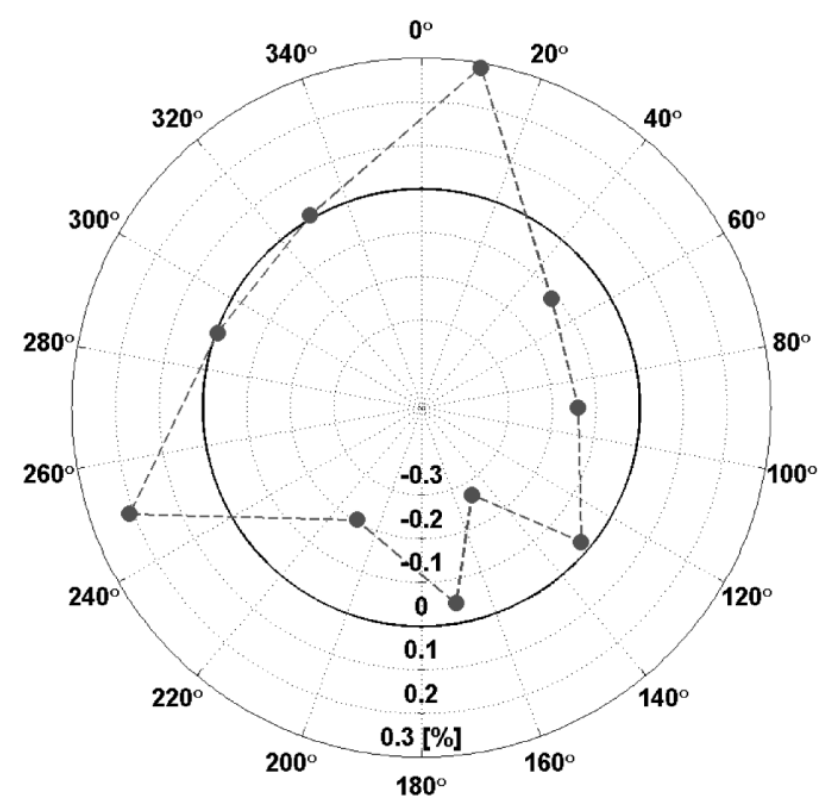

Figure 8. Polar distribution of the relative difference of $P_{01}$ local value with respect to the mean.

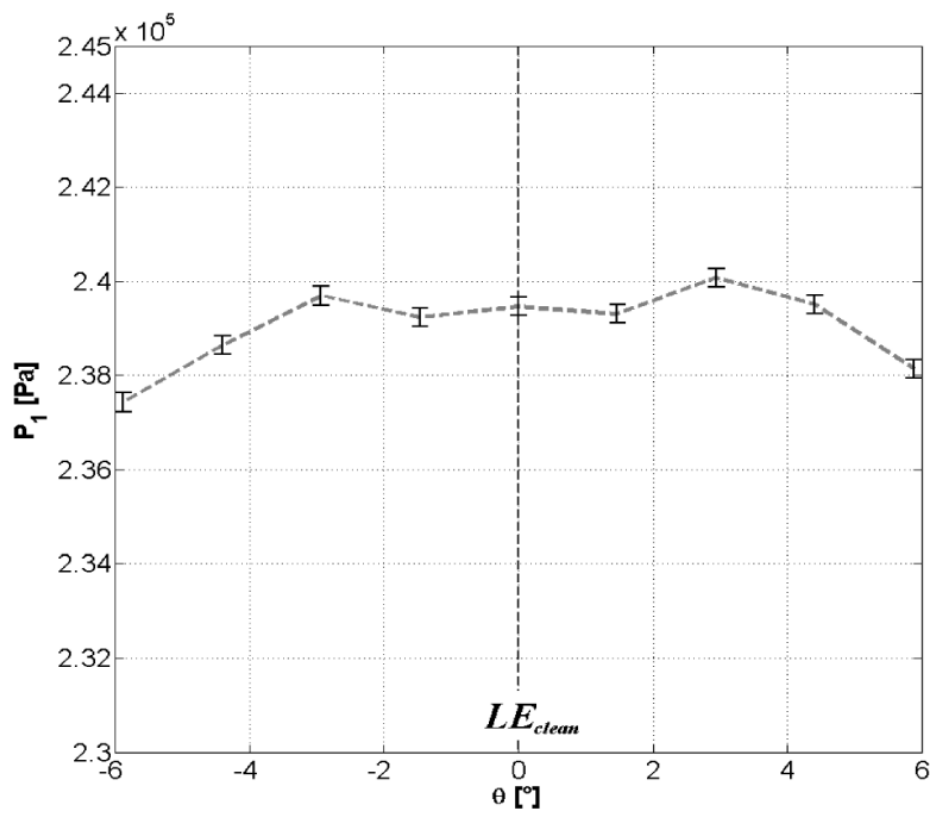

Figure 9. Inlet static pressure distribution in the trapezoidal upstream volume.

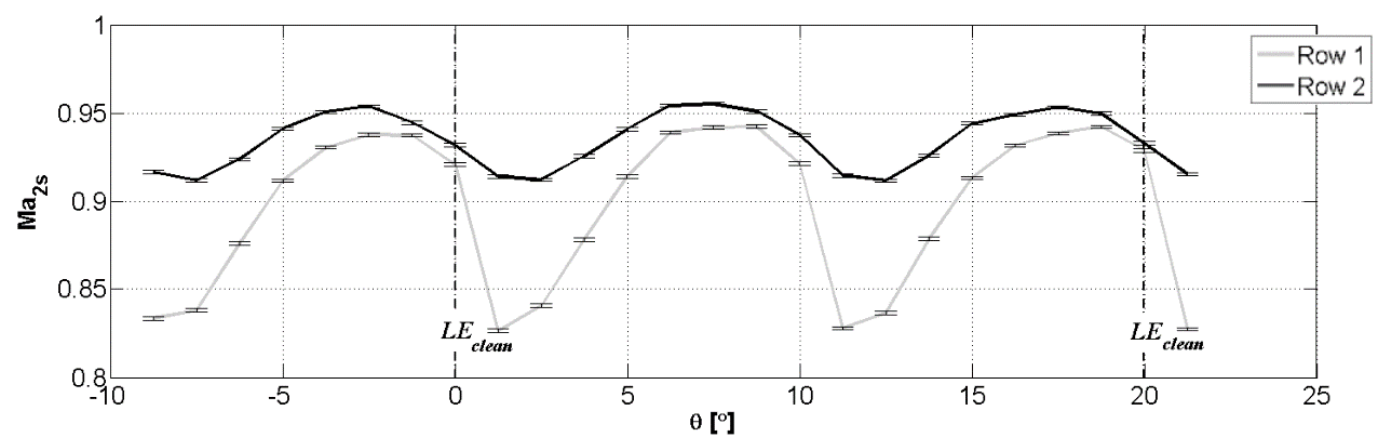

Figure 10. Isentropic downstream Mach number distribution on row 1 and row 2. 


\section{PIV Results}

The contour plots shown in Figure 11 report the velocity field (a) and the flow angle distribution (b) measured in the frame of the PIV campaign. The quantities are plotted with respect to the local polar coordinates; $\mathrm{R}$ is the radial distance from the center of the test section and $\vartheta$ the azimuthal shift from the leading edge of a "covered" blade located in 0 . On the right hand side of the figure (c,d), the computed uncertainties are provided as a percentage of the local measured value.
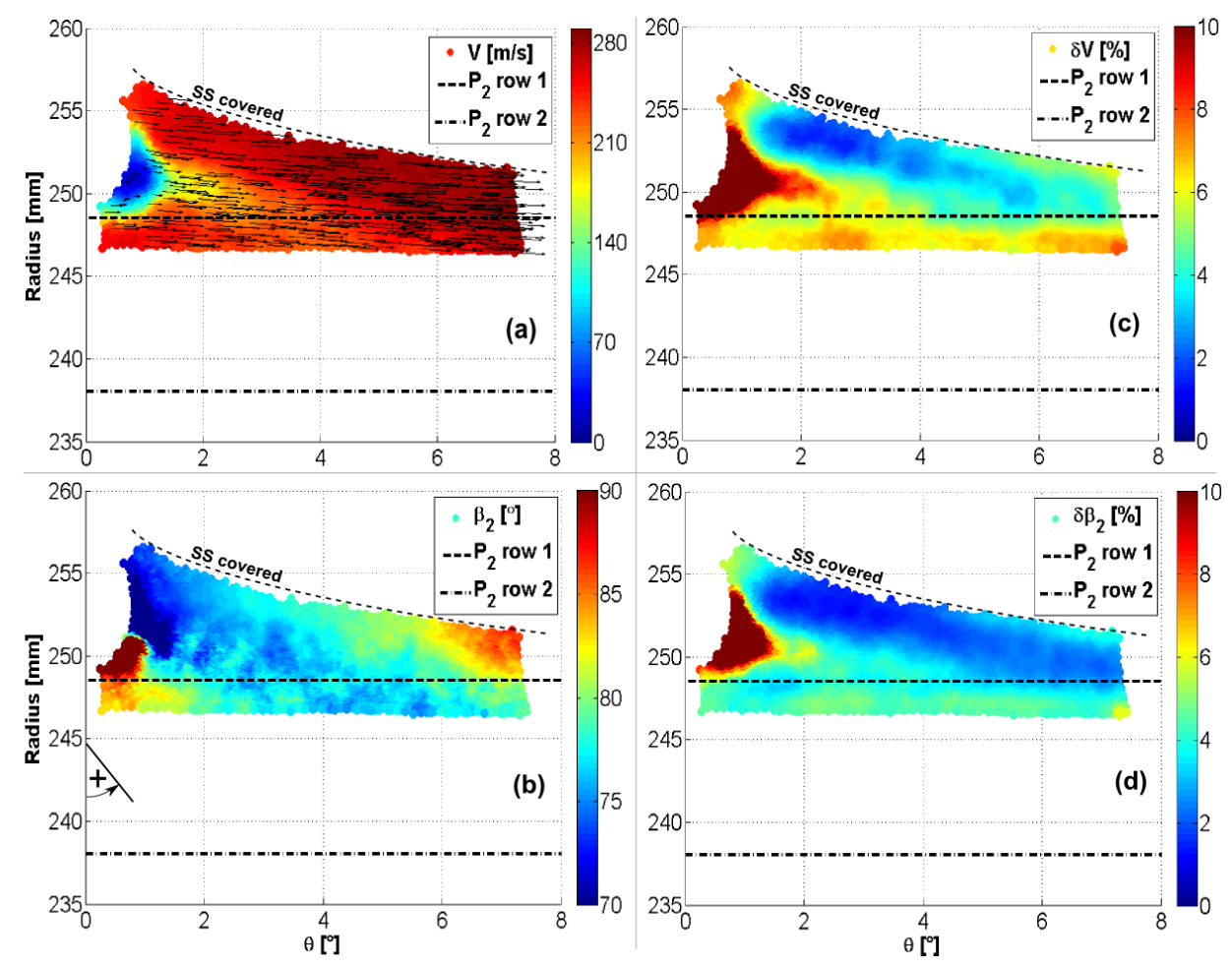

Figure 11. PIV measurement results: (a) absolute velocity (V); (b) flow angle $\left(\beta_{2}\right)$; (c) absolute velocity uncertainty $(\delta \mathrm{V})$ and $(\mathbf{d})$ flow angle uncertainty $\left(\delta \beta_{2}\right)$. Velocity vectors were under-sampled by a factor 10 .

In general, the flow field is characterized by a high velocity level, specifically in the throat region, all along the suction side of the covered blade and at the bottom, at smaller radii. From the trailing edge of the adjacent vane, the velocity deficit in the wake extends till $\vartheta=4^{\circ}$ where it is then mixed out by the upper and the lower high momentum flow regions. The velocity increases to values as high as $270 \mathrm{~m} / \mathrm{s}$. From $\vartheta=5^{\circ}$ until the end of the field of view at $\vartheta=7.3^{\circ}$, the trace of the wake cannot be appreciated anymore, with high velocities at every radial coordinate. The latter part of the flow field directly feeds the next sector, generating the high momentum region under the wake at smaller radii.

The absolute velocity contour plot described up to this point is necessarily compatible with a strong swirled behavior of the flow field as confirmed by the flow angle distribution whose magnitude remains higher than $75^{\circ}$ almost everywhere in the investigated area. In any case, $\beta_{2}$ is always smaller than $\pi / 2$, meaning that the stream tubes would show the tendency to reduce their radial coordinate while flowing clockwise. By reducing the radial coordinate, the absolute velocity is necessarily boosted by the conservation of the angular momentum, therefore, imposing a further acceleration which justifies the higher $\mathrm{Ma}_{2 \mathrm{~s}}$ measured on the second row of static taps than on row 1 (see Figure 10).

Measurement uncertainties were computed by taking into account the local standard deviation $\left(\sigma_{x}\right)$ and the number of recorded images $\left(N_{\text {records }}\right)$, as described by Equation (1) where $Z a$ is the 95 th percentile of the standard normal distribution (95\% confidence interval). Both $\delta \mathrm{V}$ and $\delta \beta_{2}$, shown in Figure 11c,d, respectively, present the highest uncertainties in the wake region of the "clean" blade. 
In fact, under the effect of the centrifugal forces generated by the von Karman street from the trailing edge, particles were concentrated at its sides, weakening the signal in its center while strengthening it close to the suction side and at smaller radial coordinates. Furthermore, except for the trailing edge region, uncertainties stay below $8 \%$ for both the absolute velocity and the flow angle.

$$
\delta x=Z a \cdot \frac{\sigma_{x}}{\sqrt{N_{\text {records }}}}
$$

\section{Pressure Probe Results}

Figure 12a reports the sector total pressure wakes $\left(\mathrm{P}_{02} / \mathrm{P}_{01}\right)$ measured by means of the 16 micro virtual 3-hole probes. The retrieved distribution was cloned over two sectors to allow a clear interpretation. As suggested by the PIV campaign results, the extreme swirling of the flow had the tendency to turn the stream tubes towards the tangential direction, leading to a more compact radial extension and to an enhancement of the mixing-out of the flow field which, in turn, led to the inability to univocally distinguish between the "clean" and the "covered" wake. Anyway, a marked asymmetry can be recognized between the high total pressure regions centered respectively at $\vartheta=11.25^{\circ}$ and $\vartheta=21.25^{\circ}$, where the centers of the blade channels were most probably located. In general, peak values keep noticeably below 1 , suggesting therefore a poor aerodynamic efficiency of the cascade which has to be entirely related to its very low aspect ratio.
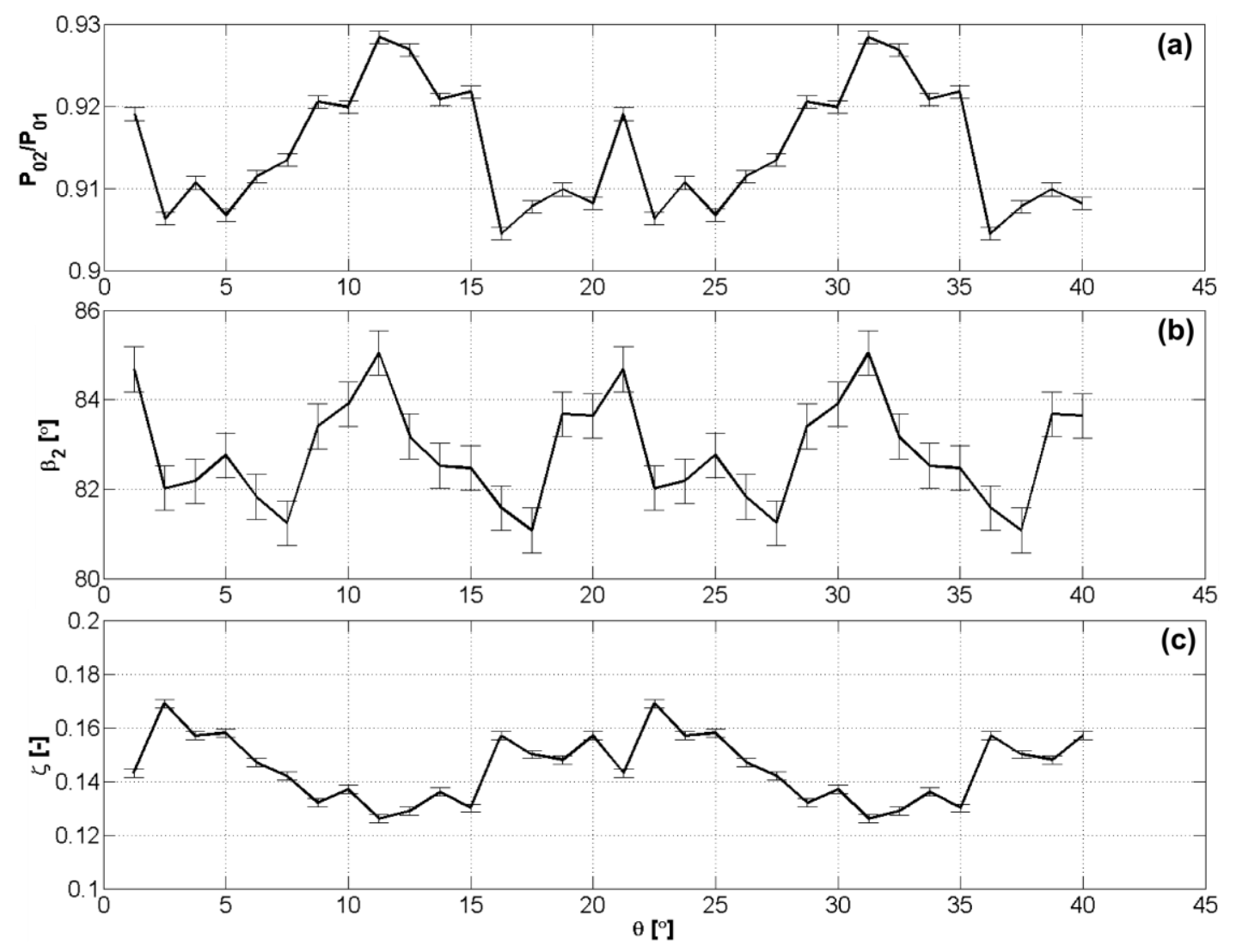

Figure 12. Micro virtual 3-hole probe results: (a) total pressure wakes $\left(\mathrm{P}_{02} / \mathrm{P}_{01}\right)$; (b) flow angle taken from the radial direction $\left(\beta_{2}\right)$ and $(\mathbf{c})$ kinetic energy loss coefficient $(\zeta)$.

The high pressure regions identified in the previous plot coincide with the peak values of the downstream flow angle. Its distribution is shown in Figure $12 \mathrm{~b}$ and it is characterized by a much more pronounced periodicity with the wakes clearly located at $\vartheta=7.5^{\circ}$ and $\vartheta=17.5^{\circ}$, respectively. The flow 
angle magnitudes describe a highly tangential flow all over the sector, with a variability limited to less than $4^{\circ}$ between peaks and valleys.

The kinetic energy loss coefficient distribution is finally presented in Figure $12 \mathrm{c}$ as defined by Equation (2), being $\gamma$ the specific heat ratio. It shows a definitely more homogeneous distribution over the sector with minima located in correspondence of the pressure peaks and of the high turning regions. The rather high loss values, always higher than $12 \%$, are a further demonstration of how important the effect of the aspect ratio is on the global performance of the cascade.

$$
\zeta=1-\frac{1-\left(\frac{P_{2}}{P_{02}}\right)^{\frac{\gamma-1}{\gamma}}}{1-\left(\frac{P_{2}}{P_{01}}\right)^{\frac{\gamma-1}{\gamma}}}
$$

In order to reduce, as much as possible, the uncertainty on pressure measurements, small-scale differential transducers were adopted and referenced to the upstream total pressure. The latter, instead, was acquired by a pressure scanner with respect to the atmospheric pressure. Such an arrangement of the measurement devices produced an uncertainty of about $26 \mathrm{~Pa}$ on probe readings and of $200 \mathrm{~Pa}$ for $\mathrm{P}_{01}$ which corresponds, in fact, to the highest contribution in the loss coefficient propagated error. In the case of the flow angle, the most important source of uncertainty was found to directly depend on the absolute probe positioning and it was estimated to be equal to $0.5^{\circ}$.

\section{Particle Image Velocimetry and Probes Measurements Comparisons}

A comparison is presented in terms of flow angle and velocity components between the pressure probes measurements and the PIV data extracted in correspondence of the first row of wall static pressure taps $\left(8.6 \% \mathrm{C}_{\mathrm{r}}\right)$. Some assumptions had to be made in order to retrieve the velocity profiles from the probe results, assumptions which are worth critical revision.

As previously mentioned, pressure probe measurements were carried out exactly at the location of the second row of wall static pressure taps $\left(44.5 \% \mathrm{C}_{\mathrm{r}}\right)$, which actually allowed the computation of a local Mach number $\left(\mathrm{Ma}_{2}\right)$ under the assumption of a homogeneous span-wise distribution of $\mathrm{P}_{2}$-row 2 . Even though the latter hypothesis had to be necessarily accepted, it also corresponded to a very crude approximation of the problem: at the measurement location, in fact, an opening is present (see Figure 2) which increases the test section span of about $20 \%$, naturally inducing important pressure gradients. Once the local Mach number was computed, a local temperature value had to be imposed to retrieve the velocity vector. In the present application, it was chosen to consider the test section as adiabatic and to impose the downstream total temperature equal to $\mathrm{T}_{01}$. This last assumption, much less drastic than the preceding one, was supported by the fact that the drop in total inlet temperature (typical of blow-down facilities) was small enough to lead to negligible heat transfer phenomena between the test section and the surrounding environment.

As previously commented in the PIV results discussion, the reduction of the radial coordinate at which the flow is flowing, necessarily implies an increase of the velocity level as an effect of the conservation of the angular momentum which, in turn, should lead to a rising magnitude of both the velocity components. This is verified for the tangential velocity $V_{\text {tg }}$ (Figure 13a) which was globally boosted all across the sector span when moving from row 1 to row 2, with a very marked increase (about 25\%) especially in the center of the wake.

On the contrary, as shown in Figure 13b, a 30\% reduction of the radial velocity component $\left(\mathrm{V}_{\mathrm{rad}}\right)$ was retrieved between the two measurement points. This result, in contrast with what was stated before, has to be related to the presence of the test section opening which augmented the flow cross-section of about $20 \%$. As a consequence, $\beta_{2}$, presented in Figure 13c, became more tangential when flowing further downstream, with a general increase of about $6^{\circ}$ all across the sector. 

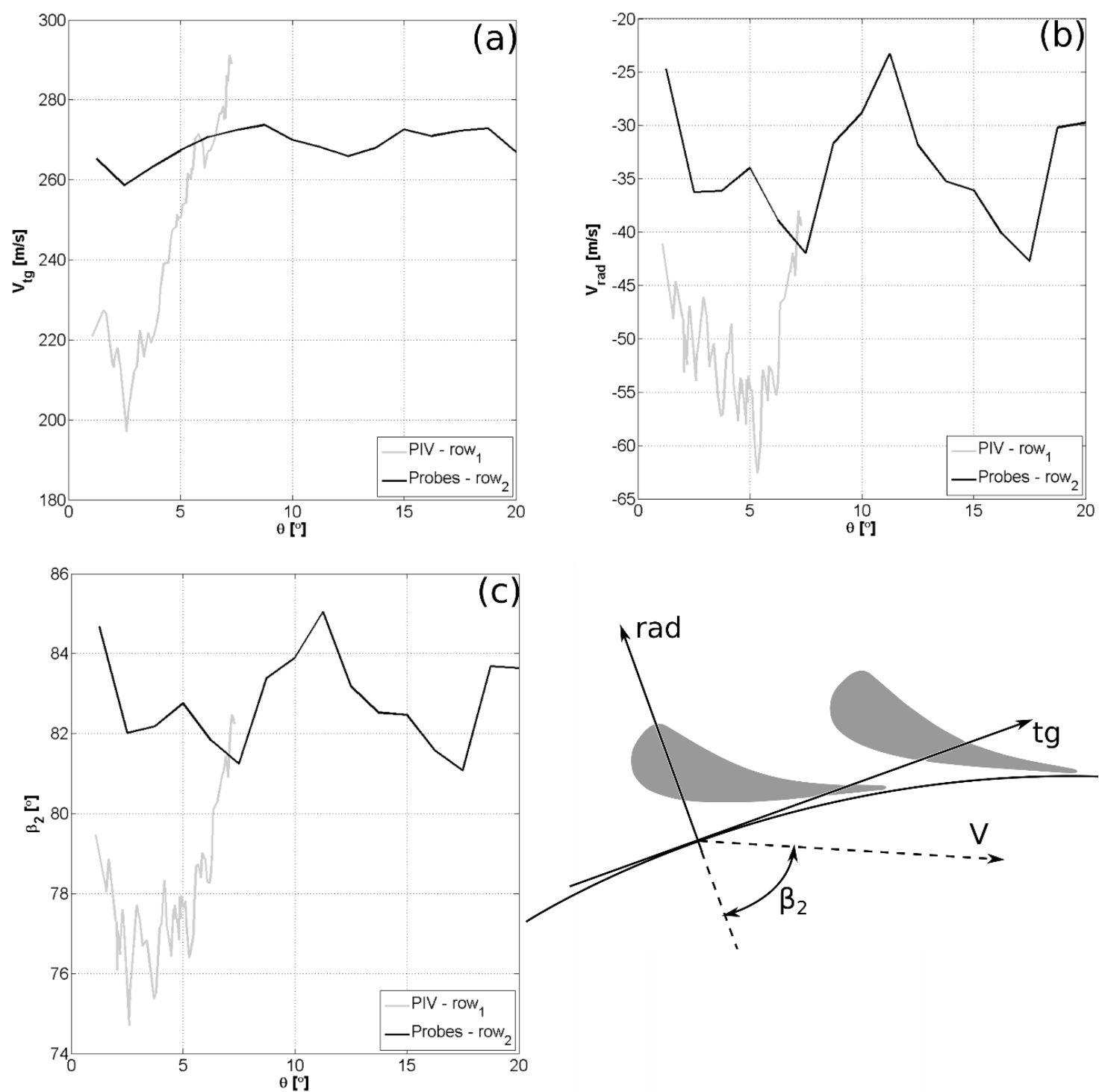

Figure 13. Comparison between PIV measurements extracted at $8.6 \%$ radial chord $\left(\mathrm{C}_{\mathrm{r}}\right)$ and probes measurements $\left(44.5 \% \mathrm{C}_{\mathrm{r}}\right)$ in terms of tangential velocity component $\left(\mathrm{V}_{\mathrm{tg}}\right)(\mathbf{a})$; radial velocity component $\left(\mathrm{V}_{\mathrm{rad}}\right)(\mathbf{b})$; and flow angle $\left(\beta_{2}\right)(\mathbf{c})$. Conventions are reported in the bottom right.

\section{Conclusions}

An extensive measurement campaign was carried out at VKI with the intent of characterizing the aerodynamic performance of an ultra-low aspect ratio centripetal turbine stator. To the authors' best knowledge, this is the first time that such a design has been experimentally investigated and described at this level of detail.

Two types of measurement techniques were used: PIV was carried out immediately downstream of the trailing edge circumference, while micro cylindrical pressure probes were operated in virtual 3-hole mode further downstream, where the test section presented an opening in the span-wise direction. The results describe a strongly swirling flow field whose tangential velocity increased when flowing towards smaller radii. The radial velocity component had exactly the opposite behavior: further downstream, in correspondence with the span-wise aperture, $\mathrm{V}_{\text {rad }}$ experienced a drastic $30 \%$ reduction to balance the bigger flow cross-section. Furthermore, the absolute velocity grew globally towards the center of the centripetal cascade. 
In general, this component showed a quite poor aerodynamic performance with kinetic energy loss levels as high as $16 \%$ which have to be completely related to the extremely small aspect ratio.

Acknowledgments: The authors wish to express their gratitude to Safran Aircraft Engines for the financial support to the measurement campaign and to Tamas Regert and to Maria Rosaria Vetrano for their precious help in the building of the PIV setup.

Author Contributions: In the frame of the present investigation, Fabrizio Fontaneto performed the full measurement campaign and data processing. Tony Arts supervised the experimental activity and provided support both during the measurement campaign and the results interpretation. Matthieu Simon and Philippe Picot performed the design of the test item.

Conflicts of Interest: The authors declare no conflict of interest.

\section{Nomenclature}

$\begin{array}{ll}\mathrm{C}_{\mathrm{r}} & \text { Radial chord }(\mathrm{mm}) \\ \mathrm{d} & \text { Diameter }(\mathrm{mm}) \\ \mathrm{Ma} & \text { Mach number }(-) \\ \mathrm{Re} & \text { Reynolds number }(-) \\ \mathrm{P} & \text { Pressure }(\mathrm{Pa}) \\ \mathrm{R} & \text { Radius }(\mathrm{mm}) \\ \mathrm{T} & \text { Temperature }(\mathrm{K}) \\ \mathrm{V} & \text { Velocity }(\mathrm{m} / \mathrm{s})\end{array}$

\section{Greek letters}

$\begin{array}{ll}\beta & \text { Flow angle }\left(^{\circ}\right) \\ \delta & \text { Measurement uncertainty }(-) \\ \vartheta & \text { Angular coordinate }\left(^{\circ}\right) \\ \zeta & \text { Kinetic energy loss coefficient }(-)\end{array}$

\section{Subscripts}

$\begin{array}{ll}0 & \text { Total conditions } \\ 1 & \text { Inlet section } \\ 2 & \text { Outlet section } \\ \mathrm{rad} & \text { Radial component } \\ \mathrm{s} & \text { Isentropic } \\ \mathrm{tg} & \text { Tangential component }\end{array}$

\section{Acronyms}

$\begin{array}{ll}\text { PIV } & \text { Particle Image Velocimetry } \\ \text { VKI } & \text { von Karman Institute for Fluid Dynamics } \\ \text { LE } & \text { Leading edge } \\ \text { TE } & \text { Trailing edge }\end{array}$

\section{References}

1. Simpson, A.T.; Spence, S.W.; Watterson, J.K. Numerical and experimental study of the performance effects of varying vaneless space and vane solidity in radial turbine stators. J. Turbomach. 2013, 135, 420-431. [CrossRef]

2. Dupont, P.; Piponniau, S.; Sidorenko, A.; Debiève, J.F. Investigation by particle image velocimetry measurements of oblique shock reflection with separation. AIAA J. 2008, 46, 1365-1370. [CrossRef] 
3. Fontaneto, F.; Arts, T.; Regert, T.; Simon, M. Experimental methodology for the performance assessment of a transonic ultra-low aspect ratio centripetal turbine stator. In Proceedings of the XXII Symposium on Measuring Techniques in Turbomachinery, Lyon, France, 4-5 September 2014.

4. Theunissen, R. Adaptive Image Interrogation for PIV: Application to Compressible Flows and Interfaces; Delft University of Technology: Delft, The Netherlands, 2010.

5. Bergh, H.; Tijdeman, H. Theoretical and Experimental Results for the Dynamic Response of Pressure Measuring Systems; Nationaal Lucht-en Ruimtevaartlaboratorium: Amsterdam, The Netherlands, 1965.

(c) 2016 by the authors; licensee MDPI, Basel, Switzerland. This article is an open access article distributed under the terms and conditions of the Creative Commons Attribution (CC-BY-NC-ND) license (https://creativecommons.org/licenses/by-nc-nd/4.0/). 